\title{
FRACTAL-LIKE BEHAVIOUR OF THE BCC/FCC PHASE SEPARATION IN THE IRON-GOLD ALLOYS
}

\author{
A. Błachowski ${ }^{1}$, K. Ruebenbauer ${ }^{1^{*}}$, A. Rakowska ${ }^{2,4}$ and S. Kąc ${ }^{3}$ \\ ${ }^{1}$ Mössbauer Spectroscopy Division, Institute of Physics, Pedagogical University \\ PL-30-084 Kraków, ul. Podchorażych 2, Poland \\ ${ }^{2}$ Faculty of Materials Science and Ceramics, AGH University of Science and Technology \\ ${ }^{3}$ Department of Surface Engineering and Materials Characterisation, Faculty of Metals \\ Engineering and Industrial Computer Science, AGH University of Science and Technology \\ PL-30-059 Kraków, Al. Mickiewicza 30, Poland \\ ${ }^{4}$ Institute of Metallurgy and Materials Science, Polish Academy of Science \\ PL-30-059 Kraków, ul. Reymonta 25, Poland
}

*Corresponding author: sfrueben@,cyf-kr.edu.pl

Keywords: iron-gold alloys, phase separation, nano-particles, fractals

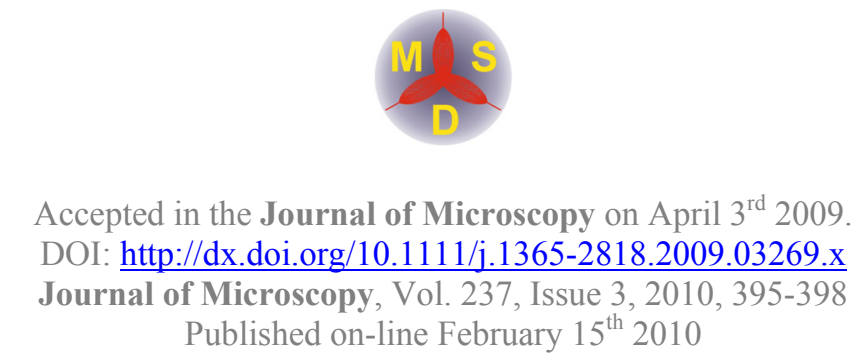

\begin{abstract}
Iron-gold alloys with compositions $\mathrm{Fe}_{70} \mathrm{Au}_{30}$ and $\mathrm{Fe}_{50} \mathrm{Au}_{50}$ were prepared by arc melting. The alloys were investigated by means of the high resolution scanning electron microscopy (SEMFEG) in the as-cast state and upon annealing in two steps, i.e., at $250{ }^{\circ} \mathrm{C}$ for $24 \mathrm{~h}$ and subsequently at $500{ }^{\circ} \mathrm{C}$ for $48 \mathrm{~h}$. The alloys were composed of two phases, i.e., a BCC phase rich in iron and a FCC phase rich in gold. The single-phase regions have equivalent diameter of about $50 \mathrm{~nm}$. SEM images show self-similar structure for the spatial distribution of the above phases on scales ranging from about $1 \mathrm{~mm}$ till about $100 \mathrm{~nm}$. The roughness of the images has been used to estimate a fractal dimension of the phase mixture. For larger scales of the as-cast samples one finds fractal dimension of about 1.7 for $\mathrm{Fe}_{70} \mathrm{Au}_{30}$ composition, i.e., very close to the dimension of typical diffusion limited aggregation (DLA) fractals. For annealed samples dimension 1.1 was found.
\end{abstract}




\section{Introduction}

The iron-gold phase diagram is quite simple (Massalski, 1990). There are no intermetallic compounds, and only two phases are possible, i.e., either the BCC phase rich in iron with very limited substitutional gold solubility (up to about 3 at.\% frozen from high temperature), or the FCC phase rich in gold with much larger substitutional iron solubility (up to about 30 at.\% retained from high temperature) (Massalski, 1990; Błachowski et al., 2008). Both phases are metastable at room temperature. The $\mathrm{BCC}$ phase is ferromagnetic at room temperature, while the FCC phase is either ferromagnetic or paramagnetic at room temperature depending upon the iron content (Coles et al., 1978; Borg \& Violet, 1987). During annealing at relatively low temperature gold moves out of the BCC phase leaving behind almost pure iron (Błachowski et al., 2008), and iron gets out of the FCC phase leaving almost pure diamagnetic gold. It was found that iron diluted in the gold FCC phase tends to make atomic clusters of various sizes at intermediate temperature (Yoshida et al., 1989). These clusters are likely to be nucleation and growth centres for the almost pure iron BCC phase. Mixed phase samples containing BCC and FCC phase could be easily made by e.g. arc melting of the elements.

It has been found that as-cast samples contain complicated regions with varying iron content. These regions are composed of both phases in varying proportions. The image changes upon sample annealing, albeit some iron rich regions are preserved for relatively low annealing temperatures applied for not too long time periods (Błachowski et al., 2008).

Fractal structures are quite common for two-phase systems, particularly if they are rapidly quenched into the metastable state, far from equilibrium (Skrotzki, 1991; Long et al., 1993; Schuszter et al., 2003). Hence, we have attempted to make an analysis of the scanning electron microscopy (SEM) images in order to look upon fractal dimension versus scale and sample annealing, i.e., the image magnification and annealing heat treatment applied.

\section{Experimental}

Samples of about $1.5 \mathrm{~g}$ were made by arc melting of the appropriate amounts of iron having $99.97+$ at.\% purity and gold of 99.96 at.\% purity under high purity argon atmosphere. The samples were remelted three times without turning them over to assure global homogeneity. Specimens of the following compositions were used in further investigations: $\mathrm{Fe}_{70} \mathrm{Au}_{30}$ and $\mathrm{Fe}_{50} \mathrm{Au}_{50}$. The exact compositions were determined by means of the electron microprobe (EDS - energy dispersion X-ray spectroscopy) averaging over the large area. Annealing was performed under vacuum in two steps at $250{ }^{\circ} \mathrm{C}$ for $24 \mathrm{~h}$ and subsequently at $500{ }^{\circ} \mathrm{C}$ for $48 \mathrm{~h}$. Phase analysis was performed using X-ray diffraction and Mössbauer spectroscopy (Błachowski et al., 2008). It was found by the X-ray diffraction that crystallites of both phases have approximate average equivalent diameter of $50 \mathrm{~nm}$, even after annealing (Błachowski et al., 2008).

The flat sample surface resulting from the preparation of the Mössbauer absorber (Błachowski et al., 2008) was polished without any further chemical treatment. An ultra high-resolution scanning electron microscope, Nova 200 NanoSEM, was used with either $15 \mathrm{keV}$ or $18 \mathrm{keV}$ primary electrons energy. A field emission gun (FEG) was used as the source of primary electrons. Back-scattered electron images were recorded at various magnifications ranging from 200x till 300000x. Back-scattered electron images were recorded to obtain maximum possible range of magnification scales, i.e., to obtain sharp images at large and small magnifications. For smaller magnifications the electron microprobe results were correlated with the darkness of the 
particular part of the image (Błachowski et al., 2008), and it has been found that indeed the iron rich regions are dark, while the gold rich regions are white. On the $1 \mu \mathrm{m}$ scale (the EDS primary electron beam diameter) one finds 14 at. $\%$ of gold in dark regions and 45 at. $\%$ of gold in bright regions for the $\mathrm{Fe}_{70} \mathrm{Au}_{30}$ sample. Corresponding values for annealed $\mathrm{Fe}_{50} \mathrm{Au}_{50}$ sample are 19 at.\% and 75 at.\%, respectively. Therefore the images represent the relative distribution of gold and iron with negligible topography effects. Image data were pixelised into square pixels with 8 bits per pixel representing linear grey scale with the highest value representing "white". Images were collected with 2048 pixels vertical and 2375 pixels horizontal.

\section{Data analysis}

Many methods used to estimate parameters essential in calculation of the eventual fractal dimension are simply useless for the photographic-like images without sharply defined borders of the regions with different brightness (Liebovitch \& Toth, 1989). We have chosen to estimate the roughness of the acquired images, as roughness could be reliably calculated even for very poorly defined images, i.e., fuzzy images like SEM images at very large magnifications (Zubimendi et al., 1994). Roughness could represent various physical properties of the real object e.g. topography, atomic composition, magnetic moment variation and many other. In our case roughness represents atomic compositional contrast.

Let us consider a planar rectangular area divided into $N \geq 3$ rows of equal height and $M \geq 3$ columns having all equal width, the latter being the same as the height of the row. Each element of this area (pixel) has a square shape with some definite (positive) size of the edge. It could be uniquely located by two indices $n=1,2, \ldots, N$ and $m=1,2, \ldots, M$. In our case $N=2048$ and $M=2375$. Each pixel is characterised by some value of the grey scale varying between $\left[0,2^{L}-1\right]$ with $L \geq 1$ standing for the number of bits per pixel, the latter being the same for all pixels with the same order and meaning of all bits for each pixel. In our case $L=8$ and therefore 256 levels of the grey scale are defined. The lowest possible value of the grey scale represents "black", while the highest possible value represents "white". Roughness $R$ could be defined in various ways, but the simplest definition relies upon differences between grey scale value of the particular pixel considered $x_{n m}$, and the grey scale value of the four adjacent pixels $x_{i}$ (i.e. $\left.x_{1}=x_{n-1, m}, x_{2}=x_{n+1, m}, x_{3}=x_{n, m-1}, x_{4}=x_{n, m+1}\right)$. Adjacent pixels are those having common edge with the considered pixel. Therefore roughness could be expressed as follows (Błachowski \& Ruebenbauer, 2009):

$$
R=[4(N-2)(M-2)]^{-1} \sum_{n=2}^{N-1} \sum_{m=2}^{M-1} \sum_{i=1}^{4}\left(\frac{x_{n m}-x_{i}}{2^{L}-1}\right)^{2} .
$$

Roughness defined in such a way satisfies the following condition $0 \leq R \leq 1$. It equals zero for completely smooth area and equals one for black and white chessboard-like pattern.

We have calculated the roughness for various images collected at various scales (magnifications) using SEM. One can plot roughness versus size of the pixel edge $r>0$ on the log-log scale. The above plot is often called Richardson plot (Mandelbrot, 1982). If one has linear regions within this plot one can define the slope $\alpha$ of the straight line within this region. The fractal dimension $d$ could be related to this slope within this region as $d=2-|\alpha|$ provided $|\alpha|<2$ (Mandelbrot, 1982, 1985). A similar method has been used previously for the STM images (Zubimendi et al., 1994). 


\section{Discussion of results}

Selected SEM images are shown in Figs 1, 2. Whiteness of the images is proportional here to the current of the back-scattered electrons. Dark areas represent the low average atomic number (iron rich regions) and vice versa. The large difference of the atomic numbers between iron and gold assures very high contrast between iron rich and gold rich regions.

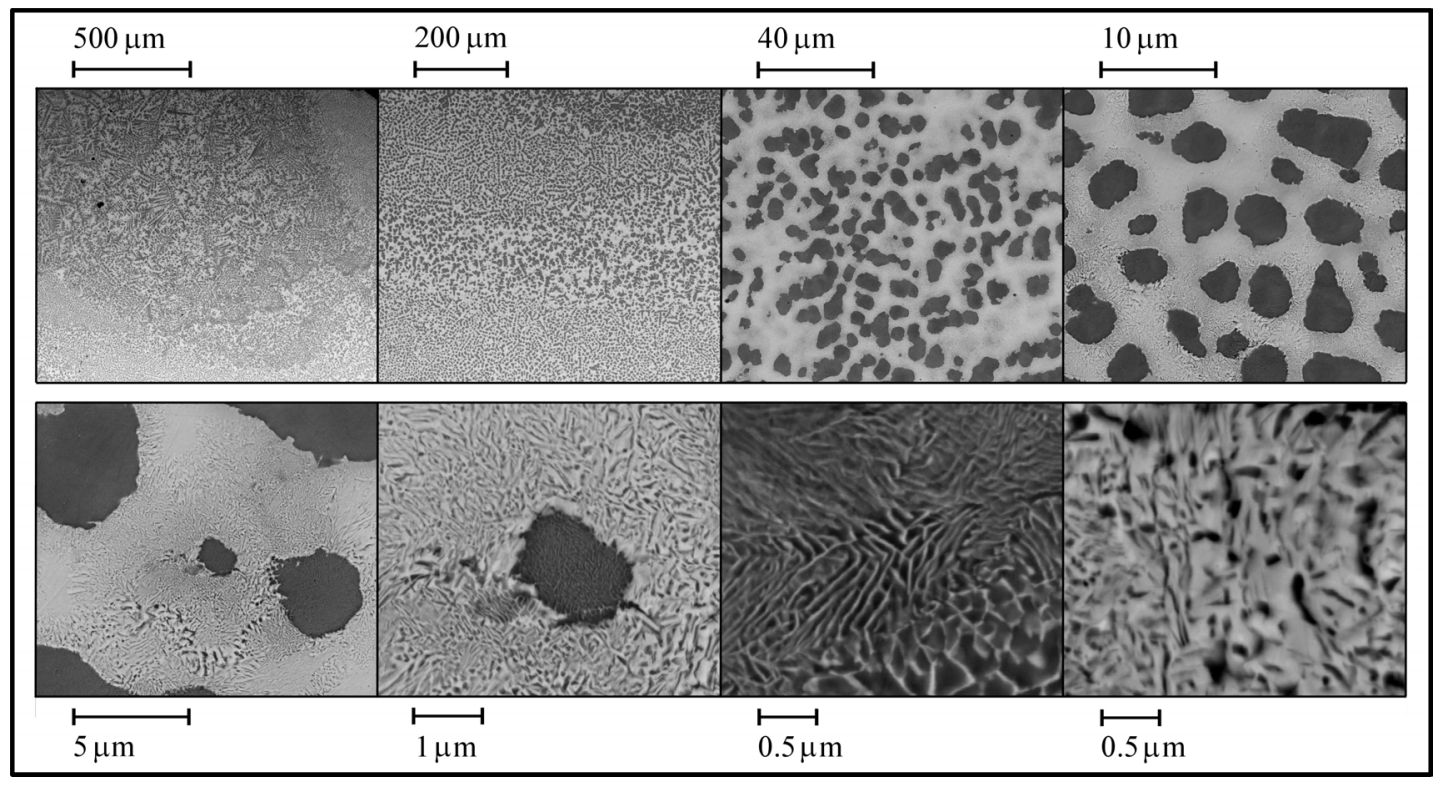

Figure 1. Examples of SEM back-scattered electron images for as-cast $\mathrm{Fe}_{70} \mathrm{Au}_{30}$ sample at various magnifications. The dark image with the $0.5 \mu \mathrm{m}$ scale is taken from the iron rich region, while the corresponding bright image is taken from the gold rich region.

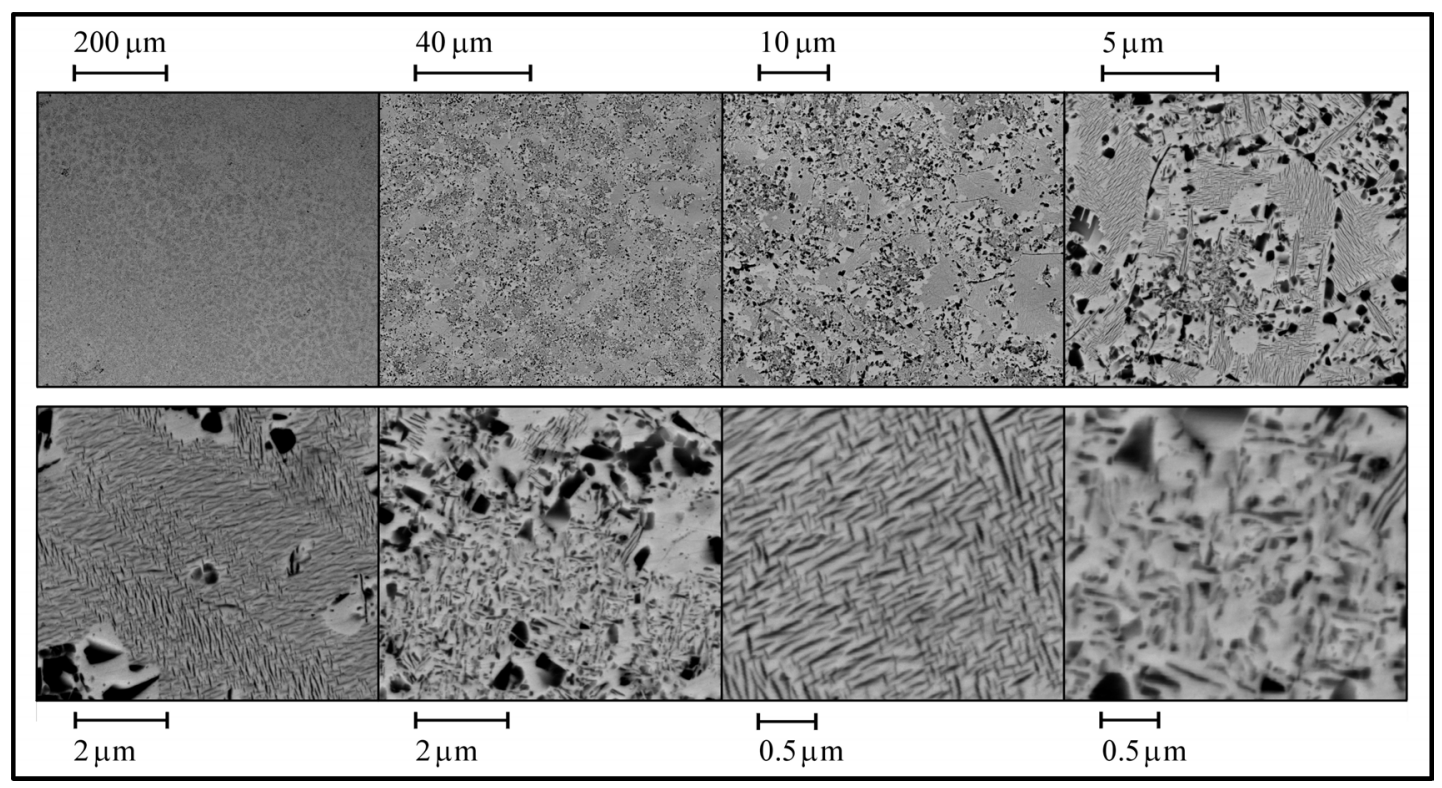

Figure 2. Examples of SEM back-scattered electron images for annealed $\mathrm{Fe}_{50} \mathrm{Au}_{50}$ sample at various magnifications. Dark and bright images with scales $2 \mu \mathrm{m}$ and $0.5 \mu \mathrm{m}$ correspond to iron rich and gold rich regions, respectively. 
Self-similarity in the statistical sense is clearly seen on the scale ranging from about $1 \mathrm{~mm}$ till about $100 \mathrm{~nm}$. It means that some fractal structure is likely to be hidden within collected images. Fig. 3 shows roughness plotted versus pixel edge size on the log-log plot for both compositions and either for the as-cast samples or for annealed samples. Each point of Fig. 3 corresponds to single image.

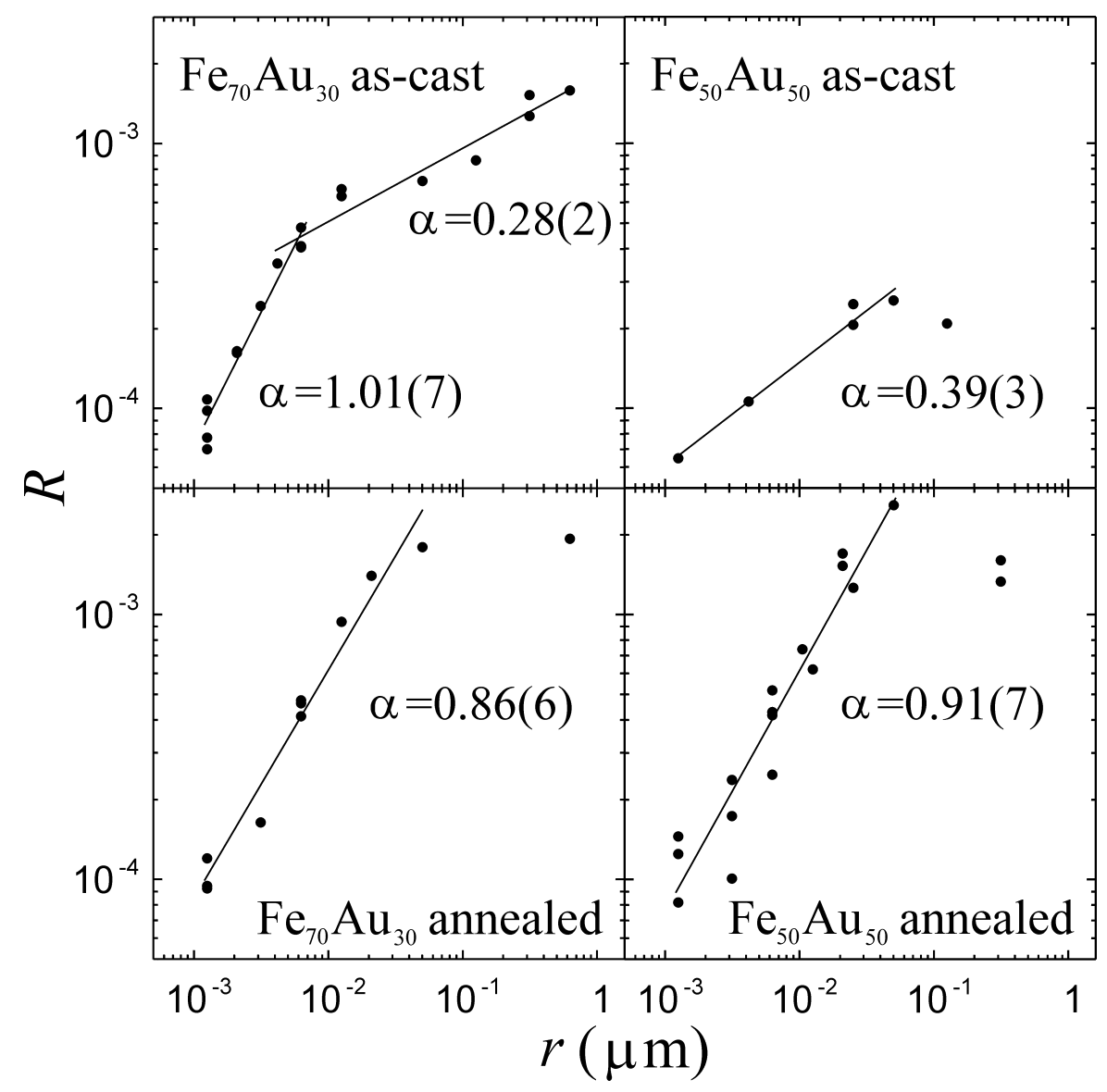

Figure 3. Richardson plots of the roughness $R$ versus pixel edge size $r$ shown for the samples investigated.

The as-cast sample $\mathrm{Fe}_{70} \mathrm{Au}_{30}$ is very similar to diffusion limited aggregation (DLA) fractal for the larger scales (smaller magnifications). DLA fractals have fractal dimension 1.71 (Witten \& Sander, 1981) in comparison with our value 1.72(2). It is believed that here operates rather hydrodynamic mechanism of DLA formation. The latter relies on mixing of two liquids with vastly different viscosities (Saffman \& Taylor, 1958; Shraiman \& Bensimon, 1984). It is well known fact that pure liquid iron has very high viscosity, while pure liquid gold is quite fluent.

On the smaller scales (large magnifications) fractal dimension of the as-cast $\mathrm{Fe}_{70} \mathrm{Au}_{30}$ sample is reduced to about unity, i.e. 0.99(7), indicating incoherence between different iron rich regions probably due to the fact that iron occurs mainly in the BCC phase. Hence, this sample could be considered as multi-scale fractal. Images of the as-cast $\mathrm{Fe}_{50} \mathrm{Au}_{50}$ sample are less contrasted as there is less BCC phase at this composition. In general this sample is characterised by unique fractal dimension 1.61(3) except for the largest scale. 
Annealed samples have fractal dimension 1.14(6) for $\mathrm{Fe}_{70} \mathrm{Au}_{30}$ composition and 1.09(7) for $\mathrm{Fe}_{50} \mathrm{Au}_{50}$ composition except for the largest scales. It means that the original coherence between iron rich regions is lost during annealing. Islands of the iron rich regions are formed, and lamellar structure is developed - particularly for the $\mathrm{Fe}_{50} \mathrm{Au}_{50}$ sample.

A fractal structure is due to the self-similar volume like regions embedded one in another and seen on the planar cross section of the image.

\section{Acknowledgement}

Dr. Jan Żukrowski, Solid State Department, Faculty of Physics and Applied Computer Science, AGH University of Science and Technology, Kraków, Poland is warmly thanked for casting and annealing iron-gold alloys used in present investigation.

\section{References}

Błachowski A. \& Ruebenbauer K. (2009) Roughness method to estimate fractal dimension, Acta Physica Polonica A 115, 636-640; see also: www.elektron.ap.krakow.pl/frct.pdf

Błachowski, A., Ruebenbauer, K., Przewoźnik, J. \& Żukrowski, J. (2008) Hyperfine interactions on iron nuclei in the BCC and fractally decomposed BCC/FCC mixed phase iron-gold alloys, $J$. Alloys Compd. 458, 96-103.

Borg, R.J. \& Violet, C.E. (1987) Resolved magnetic hyperfine spectra of Au-Fe alloys as a function of temperature, J. Phys. Chem. Solids 48, 1239-1244.

Coles, B.R., Sarkissian, B.V.B. \& Taylor, R.H. (1978) The role of finite magnetic clusters in AuFe alloys near the percolation concentration, Phil. Mag. B 37, 489-498.

Liebovitch, L.S. \& Toth, T. (1989) A fast algorithm to determine fractal dimensions by box counting, Phys. Lett. A 141, 386-390.

Long, Q.Y., Wen, Y.H., Zhu, Z., Zhang, X.M., Mu, Z.Q. \& Lung, C.W. (1993) Sierpinski fractal description of the martensitic transformation, Phil. Mag. A 68, 885-890.

Mandelbrot, B.B. (1982) The Fractal Geometry of Nature, Freeman, New York.

Mandelbrot, B.B. (1985) Self-Affine Fractals and Fractal Dimension, Phys. Scr. 32, 257-260.

Massalski, T.B. (1990) Binary Alloys Phase Diagrams, vol. 1, $2^{\text {nd }}$ ed. ASM International, p. 368. Materials Park, $\mathrm{OH}, \mathrm{USA}$.

Saffman, P.G. \& Taylor, G.I. (1958) The penetration of a fluid into a porous medium or HeleShaw cell containing a more viscous liquid, Proc. Royal Soc. (London) Ser. A 245, 312-329.

Schuszter, M., Bodnár, Z., Dobos, L. \& Mojzes, I. (2003) A novel evaluation method to determine the fractal dimension of SEM images: a study of $\mathrm{Au} / \mathrm{Pd} / \mathrm{GaAs}$ contacts during heat treatment, Phys. Stat. Sol. (c) 3, 1051-1054.

Shraiman, B. \& Bensimon, D. (1984) Singularities in nonlocal interface dynamics, Phys. Rev. A 30, 2840-2842.

Skrotzki, B. (1991) Fractal analysis of martensite in an Fe-Ni alloy, J. Materials Science 26, 1073-1077.

Witten, Jr. T.A. \& Sander, L.M. (1981) Diffusion-Limited Aggregation, a Kinetic Critical Phenomenon, Phys. Rev. Lett. 47, 1400-1403. 
Yoshida, Y., Langmayr, F., Fratzl, P. \& Vogl, G. (1989) Short-range order in Au-Fe alloys studied by high-temperature Mössbauer spectroscopy, Phys. Rev. B 39, 6395-6402.

Zubimendi, J.L., Vela, M.E., Salvarezza, R.C., Vázquez, L., Vara, J.M. \& Arvia, A.J. (1994) Fractal to nonfractal behavior of vapor-deposited gold surfaces and the relationship to the substrate temperature, Phys. Rev. E 50, 1367-1371. 